\title{
Calendar of Forthcoming Meetings Powder Diffraction June 2015
}

\author{
Gang Wang \\ Research \& Development Center for Functional Crystals, Institute of Physics, Chinese Academy of Sciences, \\ No. 8 Nansanjie, Zhongguancun, Haidian District, Beijing 100190, China \\ gangwang@iphy.ac.cn
}

28 June-2 July 2015

International Symposium on Zeolites and Microporous Crystals (ZMPC2015) Sapporo, Japan [Info: http://www. knt.co.jp/ec/2015/zmpc/index.html]

29 June-3 July 2015

International Conference on Advances in Functional Materials Stony Brook University, Long Island, New York, USA [Info: http://functionalmaterials.org/]

29 June-3 July 2015

2nd International Conference on Tomography of Materials and Structures (ICTMS2015) Quebec, Canada [Info: http://www.ictms.ugent.be/downloads/ictms2015_first_ announcement.pdf]

29 June-5 July 2015

2nd Baikal Materials Science Ulan-Ude, Russia

More information coming soon

6-10 July 2015

12th International Conference on Synchrotron Radiation Instrumentation (SRI 2015) New York City, New York, USA [Info: http://www.bnl.gov/sri2015/]

\section{9-23 July 2015}

2nd Asia-Oceania Conference on Neutron Scattering (AOCNS 2015) Sydney, Australia [Info: http://www. aocns-2015.com/]

\section{5-29 July 2015}

2015 Annual Meeting of American Crystallographic Association Philadelphia, Pennsylvania, USA [Info: http:// www.amercrystalassn.org/2015-scientific-program]

\section{6-31 July 2015}

Gordon Research Conference on X-Ray Science Easton, Massachusetts, USA [Info: http://www.grc.org/programs. aspx?id=12235]
2-6 August 2015

Microscopy \& Microanalysis 2015 Meeting Oregon Convention Center in Portland, Oregon, USA [Info: http:// www.microscopy.org/MandM/2015/]

\section{3-7 August 2015}

2015 Denver X-ray Conference (DXC) in conjunction with the 16th International Conference on Total Reflection X-ray Fluorescence Analysis and Related Methods (TXRF 2015). Westminster, Colorado, USA [Info: http:// www.dxcicdd.com/index.htm]

16-20 August 2015

XXIV International Materials Research Congress Cancun, Mexico [Info: http://www.mrs.org/imrc-2015/]

\section{3-28 August 2015}

The 29th European Crystallographic Meeting Rovinj, Croatia [Info: http://ecm29.ecanews.org/]

\section{3-28 August 2015}

16th International Conference on X-ray Absorption Fine Structure (XAFS16) Karlsruhe, Germany [Info: http:// www.xafs16.org/]

\section{3-28 August 2015}

The 11th International Conference on Materials \& Mechanisms of Superconductivity $\left(M^{2} S\right.$ 2015) Geneva, Switzerland [Info: http://www.m2s-2015.ch/]

30 August-4 September 2015

VI European Conference on Neutron Scattering (ECNS2015) Zaragoza, Spain [Info: http://ecns2015.unizar.es/]

30 August-4 September 2015

APERIODIC 2015 Břevnov Monastery, Prague, Czech Republic [Info: http://crysa.fzu.cz/aperiodic2015/] 
6-10 September 2015

International Conference on Diamond and Carbon Materials Maritim Hotel Bad Homburg, Bad Homburg, Germany [Info: http://www.diamond-conference.elsevier.com/]

6-11 September 2015

11th International Symposium on Crystalline Organic Metals, Superconductors and Magnets Bad Gögging, Germany [Info: http://www.iscom2015.de/]

\section{9-11 September 2015}

The Fifth European Conference on Crystal Growth (ECCG5) Bologna, Italy [Info: http://www.eccg5.eu/]

\section{3-18 September 2015}

16th International Conference on Small-Angle Scattering (SAS2015) Berlin, Germany [Info: http://eventegg.com/ sas-2015/]

\section{4-18 September 2015}

23rd International Congress on $\mathrm{X}$-ray Optics and Microanalysis (ICXOM23) Upton, New York, USA [Info: http://www.bnl.gov/icxom23/]

\section{0-24 September 2015}

XXIII Conference on Applied Crystallography Krynica Zdrój, Poland [Info: http://www.cac.us.edu.pl/]

\section{4-8 October 2015}

Materials Science \& Technology 2015 (MS\&T15) Greater Columbus Convention Center, Columbus, Ohio, USA [Info: http://matscitech.org/]

\section{5-29 October 2015}

2015 AAPS Annual Meeting and Exposition Orlando, Florida, USA[Info: http://www.aaps.org/annualmeeting/]
26-29 October

12th Chinese National Conference on X-ray Diffraction and ICDD Workshop Mianyang, Sichuan, China

More information coming soon

1-4 November 2015

2015 GSA Annual Meeting Baltimore, Maryland, USA [Info: http://www.geosociety.org/meetings/2015/]

29 November-4 December 2015

2015 MRS Fall Meeting \& Exhibit Boston, Massachusetts, USA [Info: http://www.mrs.org/fall2015/]

First week of December 2015

7th Joint BER II and BESSY II User Meetings Berlin, Germany [Info: http://www.helmholtz-berlin.de/user/usermeetings/index_en.html]

6-10 December 2015

4th Nano Today Conference JW Marriott Marquis Hotel Dubai, Dubai, UAE [Info: http://www.nanotodayconference.com/]

\section{4-18 March 2016}

APS March Meeting 2016 Baltimore, Maryland, USA [Info: http://www.aps.org/meetings/meeting.cfm?name=MAR16]

\section{7-22 April 2016}

116th Annual Meeting of the American Roentgen Ray Society Los Angeles, California, USA [Info: http://www. arrs.org/Education/Meetings/]

\section{August-1 September 2016}

The 30th European Crystallographic Meeting (ECM-30) Basel, Switzerland

More information coming soon 